\title{
Algorithme BSA pour les problèmes d'interaction fluide-structure
}

\section{BSA algorithm for fluid-structure interaction problems}

\author{
R. El Maani ${ }^{1}$, B. Radi², A. El Hami ${ }^{3}$ \\ ${ }^{1}$ LMN, INSA de Rouen, France, rabii.el_maani@insa-rouen.fr \\ 2LIMII, FST Settat, Maroc, bouchaib.radi@yahoo.fr \\ ${ }^{3}$ LMN, INSA de Rouen, France, abdelkhalak.elhami@insa-rouen.fr
}

RÉSUMÉ. Les problèmes d'interaction fluide-structure (IFS) jouent un rôle important dans de nombreuses applications multi-physiques comme les éoliennes, les avions, les systèmes d'injection, les vannes ou les pompes. Ainsi, l'optimisation de tels types de problèmes revêt une grande importance pratique. Les algorithmes d'optimisation visent à trouver les meilleures valeurs des paramètres d'un système sous diverses conditions. Dans ce travail une approche d'optimisation multi-objectif pour les problèmes d'IFS est présentée. elle est basée sur l'algorithme BSA (Backtracking Search Optimization), un nouveau algorithme évolutionnaire, pour résoudre des problèmes d'optimisation numérique à valeur réelle. Une procédure de couplage partitionnée est utilisée pour la simulation numérique du problème d'IFS et les résultats du front optimisé obtenu par l'algorithme BSA non-dominé sont ensuite présentés.

ABSTRACT. Fluid-structure interaction ( $\mathrm{FSI}$ ) problems play an important role in many technical applications, for instance, wind turbines, aircrafts, injection systems, or pumps. Thus, the optimization of such kind of problems is of high practical importance. Optimization algorithms aim to find the best values for a system's parameters under various conditions. In this paper an approach for multi-objective optimization of FSI problems is presented. It is based on the Backtracking Search Optimization Algorithm (BSA), a new evolutionary algorithm for solving real-valued numerical optimization problems. A partitioned coupling procedure is used for the numerical simulation of the FSI problem and the results of the optimized front obtained using non-dominated BSA algorithm are then presented.

MOTS-CLÉS. Interaction fluide-structure, optimisation multi-objectif, algorithme évolutionnaire.

KEYWORDS. Fluid-structure interaction, multi-objective optimization, evolutionary algorithm.

\section{Introduction}

Les problèmes d'interaction fluide-structure (IFS) jouent un rôle important dans de nombreuses applications multi-physiques comme les éoliennes, les avions, les systèmes d'injection, les vannes ou les pompes. Ainsi, l'optimisation de tels types de problèmes revêt une grande importance pratique. Les développements portant sur l'optimisation de forme des problèmes d'IFS restent encore limités même si beaucoup de travaux concernant les méthodes numériques de tels problèmes ont été publié ces dernières années [1, 2, 3].

L'algorithme BSA (Backtracking Search Algorithm) est un nouvel algorithme évolutionnaire, développé pour résoudre les problèmes d'optimisation numérique en ingénierie [4, 5], il utilise l'information obtenue des générations précédentes pour rechercher des solutions avec le meilleur fitness. Par définition, l'optimisation cherche la meilleure performance possible d'un modèle, il est formulé en mathématique par la minimisation d'une fonction ou d'un ensemble de fonctions en même temps et cela concerne l'optimisation simple et multi-objectif. L'utilisation des techniques de l'optimisation multi-objectif reste cruciale pour les applications réelles en raison de la multiplicité des principes impliqués. La grande majorité des applications réelles dépend de plusieurs variables du modèle donné et leur traitement de manière appropriée est la clé d'une optimisation réussie [6].

Dans cet article, on a utilisé une nouvelle méthode d'optimisation multi-objectif basée sur l'algorithme 
BSA pour minimiser le volume et la déflexion d'une plaque soumise aux forces d'un écoulement de fluide. Cet article est divisé en trois sections, dans la première on présente les équations de base du problème d'IFS ; dans la deuxième section, on introduit l'algorithme d'optimisation BSA et la troisième section présente une application numérique du BSA sur un problème d'IFS.

\section{Problème d'interaction fluide-structure}

Un problème général d'IFS consiste en la description des champs solide et fluide, des conditions de l'interface conjointe et des conditions aux limites restantes. Dans cet article, on se limite à des écoulements incompressibles, ce qui est un choix raisonnable pour de nombreuses applications d'ingénierie.

La partie fluide du problème IFS est régie par les équations de Navier-Stokes pour un écoulement incompressible qui peuvent être écrites sur le domaine spatial de la mécanique des fluides comme [7, 8] :

$$
\begin{aligned}
\frac{(\partial v)}{\partial t}+\nabla \cdot(\rho v \otimes v-\sigma)-\rho f & =0 \\
\nabla \cdot v & =0
\end{aligned}
$$

où $\rho, v$, et $f$ sont la densité, la vitesse et la force externe, et $\sigma$ le tenseur des contraintes.

L'équation régissant la réponse dynamique d'une structure ou d'un milieu élastique peut être écrite sous forme matricielle [9] comme :

$$
m \ddot{u}+c \dot{u}+k u=f(t)
$$

où $m$ est la matrice masse de la structure, $\ddot{u}$ le vecteur d'accélération, $c$ la matrice d'amortissement de la structure, $\dot{u}$ le vecteur vitesse, $k$ la matrice de rigidité de la structure, $u$ le vecteur déplacement et $f$ le vecteur des forces en fonction du temps.

Les conditions principales à l'interface sont les conditions de couplage dynamique et cinématique. L'équilibre des forces exige l'égalité des vecteurs de contraintes comme suit :

$$
\sigma^{f} . n=\sigma^{s} . n \quad \forall x \in \Gamma^{f s i}
$$

aussi l'égalité des vitesses normales à l'interface :

$$
v . n=\frac{\partial u}{\partial t} \cdot n \quad \forall x \in \Gamma^{f s i}
$$

\section{Algorithme de l'optimisation multi-objectif}

Les algorithmes d'optimisation visent à trouver les meilleures valeurs des paramètres d'un système dans des conditions diverses. La première étape dans la résolution d'un problème d'optimisation consiste à déterminer la fonction objectif qui définit les relations entre les paramètres et les contraintes du système. Pour des raisons variées, la fonction objectif peut avoir une forme non linéaire, complexe ou non différentiable. Les problèmes d'optimisation sont généralement conçus de manière à définir l'optimum global d'une fonction objectif comme étant un minimum global. 


\subsection{Optimalité de Pareto}

Considérons le problème d'optimisation multi-objectif suivant :

$$
\begin{cases}\min _{x \in \Omega} & f(x)=\left(f_{1}(x), \cdots, f_{m}(x)\right)^{T} \\ \text { s.c } & g_{i}(x) \leq 0,(i=1, \cdots, l) \\ & h_{j}(x)=0,(j=1, \cdots, k)\end{cases}
$$

où $m$ est le nombre de fonctions objectifs, $x=\left(x_{1}, \cdots, x_{n}\right) \in \Omega$ un vecteur de $n$ variables définissant l'espace de décision, $g_{i}(x)(i=1, \cdots, l)$ les $l$ contraintes d'inégalité et $h_{j}(x)(j=1, \ldots, k)$ les $k$ contraintes d'égalité.

On présente ci-dessous quelques concepts basiques de l'optimisation évolutionnaire multi-objectif [10] :

1. Pareto dominance : Soient $\mathbf{a}=\left(a_{1}, \ldots, a_{n}\right)$ et $\mathbf{b}=\left(b_{1}, \ldots, b_{n}\right)$ deux solutions différentes d'un problème d'optimisation multi-objectif. $\mathbf{a}$ domine $\mathbf{b}$ si et seulement si :

$$
\left(\forall k \in\{1, \cdots, m\} f_{k}(\mathbf{a}) \leq f_{k}(\mathbf{b})\right) \wedge\left(\exists k \in\{1, \ldots, m\}: f_{k}(\mathbf{a})<f_{k}(\mathbf{b})\right)
$$

(noté $\mathbf{a} \succ \mathbf{b})$.

2. Pareto-optimal : Une solution a est dite solution Pareto-optimale si et seulement si :

$$
\neg \exists \mathbf{x} \in \Omega \text { tel que } \mathbf{x} \succ a
$$

3. Ensemble Pareto-optimal : L'ensemble $P$ de toutes les solutions Pareto-optimales, est défini par :

$$
P=\{\mathbf{x} \mid \neg \exists \mathbf{a} \in \Omega: f(\mathbf{x}) \succ f(\mathbf{a})\}
$$

4. Front de Pareto-optimal : L'ensemble $\mathrm{P}_{F}$ comprend les valeurs de toute fonction objectif correspondant aux solutions dans :

$$
P_{F}=\left\{f(\mathbf{x})=\left(f_{1}(\mathbf{x}), \ldots, f_{m}(\mathbf{x})\right)^{T} \mid \mathbf{x} \in P\right\}
$$

\section{Optimisation en IFS}

Cette section illustre l'application de l'algorithme BSA pour un problème d'IFS traitant la déformation d'une plaque couplé à un domaine fluide.

On considère une plaque élastique en 2D soumise aux forces d'un écoulement de fluide dans un canal avec une vitesse d'entrée de $0.2 \mathrm{~m} / \mathrm{s}$. La géométrie du problème est présentée sur la figue 1 . 


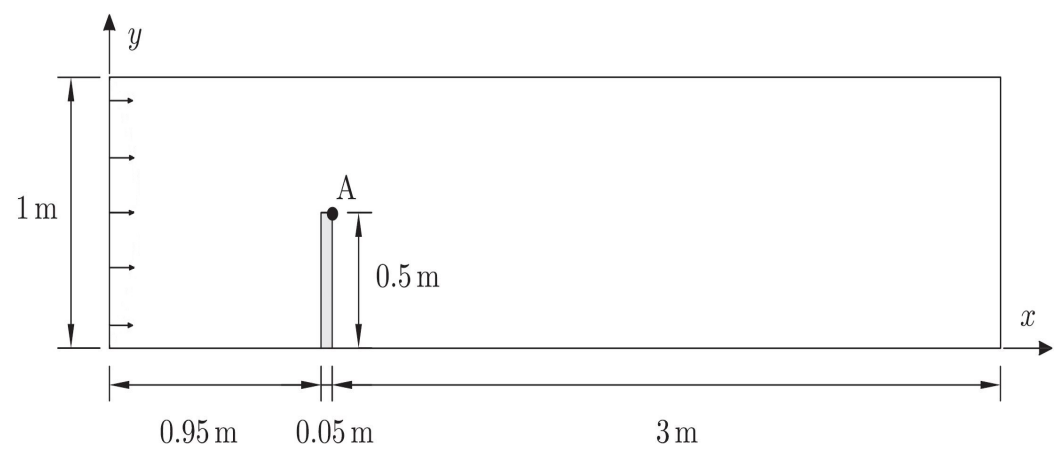

Figure 1. Géométrie et conditions aux limites du problème

Une condition de gradient nul est appliquée à la sortie et une condition de non-glissement est employée aux parois du canal et de la plaque. Les paramètres de matériaux appropriés pour le fluide et la structure sont les suivants : $\rho_{f}=1000 \mathrm{~kg} / \mathrm{m}^{3}, \mu_{f}=1 \mathrm{~kg} / \mathrm{m} . \mathrm{s}, E_{s}=5000 \mathrm{~N} / \mathrm{m}^{2}$ et $\nu_{s}=0.4$.

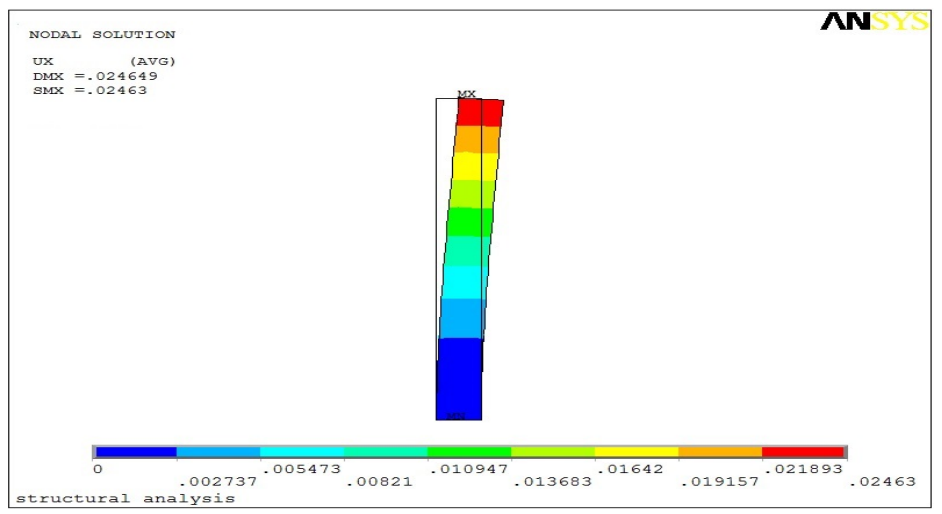

Figure 2. Déformation de la plaque

L'objectif dans ce cas d'optimisation est d'obtenir une déflexion minimale de la plaque avec un volume minimal :

$$
\min _{d \in D} f(d): \quad\left(\begin{array}{c}
u_{x}^{A} \\
V
\end{array}\right)
$$

La forme de la plaque est définie par deux splines, qui sont reliées aux faces supérieure et inférieure. La paramétrisation est réalisée avec 5 variables de conception selon la figure 3.

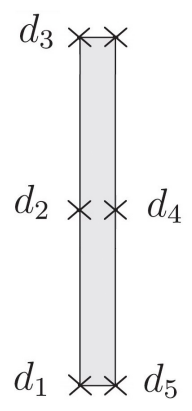

Figure 3. Paramétrisation de la plaque 


\subsection{Résultats}

La figure 5 montre le front de Pareto optimisé obtenu en utilisant l'algorithme BSA non-dominé, pour 7 itérations et une taille de population égale à 20, et le tableau 4.1 présente les valeurs minimales et maximales trouvées pour le volume et la déflexion de la plaque. Les solutions du front de Pareto sont générées et actualisées au fur et à mesure du processus d'optimisation.

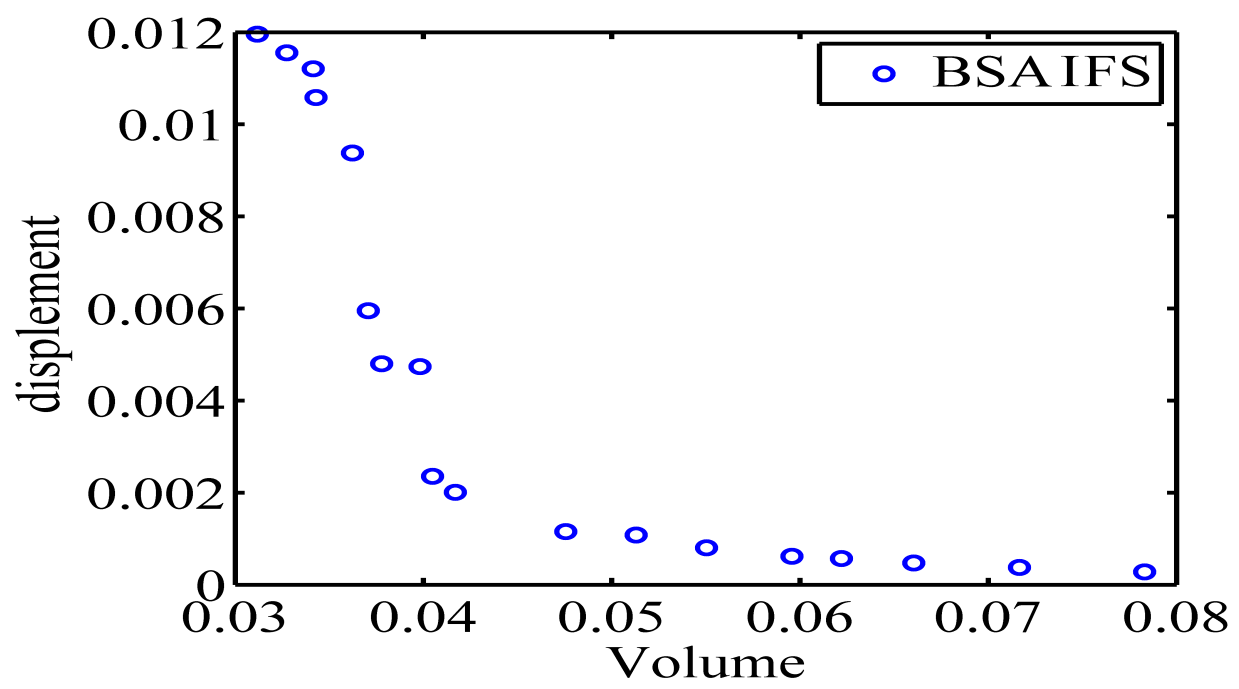

Figure 4. Front de Pareto de l'optimisation IFS

\begin{tabular}{|c|c|c|}
\hline Problème & Max & Min \\
\hline Volume & 0.0783 & 0.0312 \\
\hline Déplacement & 0.0120 & $2.772 \mathrm{e}-04$ \\
\hline
\end{tabular}

Tableau 4.1. Résultats d'optimisation

\section{Conclusion}

Cet article présente l'algorithme d'optimisation BSA, un nouvel algorithme évolutionnaire pour résoudre les problèmes d'optimisation multi-objectif, il est appliqué ici pour traiter un problème d'IFS. La structure algorithmique du BSA lui permet de bénéficier des populations de la génération précédente en utilisant des solutions trouvées précédemment pour avoir des solutions avec le meilleur fitness. L'ensemble des solutions du front de Pareto montre une efficacité et une bonne distribution. L'étude numérique a été réalisée à l'aide d'un code développé qui couple MATLAB (pour le problème d'optimisation) et ANSYS (pour le modèle éléments finis) afin d'évaluer les fonctions objectifs calculées.

\section{Remerciements}

Les auteurs tiennent à remercier "Erasmus Mundus Programme, Action 2 - STRAND 1, Lot 1, North Africa Countries" pour leur soutien financier pour la réalisation de ce travail. 


\section{Bibliographie}

[1] Maute K., Nikbay M., Farhat C., «Sensitivity analysis and design optimization of three-dimensional non-linear aeroelastic systems by the adjoint method », International Journal for Numerical Methods in Engineering, 56 : 911 933, 2003.

[2] Maute K., Allen M., «Conceptual design of aeroelastic structures by topology optimization », Structural and Multidisciplinary Optimization, $1: 27-42,2004$.

[3] Lund E., MøLler H., JAKOBSEn L., «Shape design optimization of stationary fluid-structure interaction problems with large displacements and turbulence », Structural and Multidisciplinary Optimization, 25 : 383-392, 2003.

[4] Civicioglu P., «Backtracking Search Optimization Algorithm for numerical optimization problems », Applied Mathematics and Computation, $219: 8121-8144,2013$.

[5] Oliveira A., Nedjah N., Mourelle L.M., «Genetic and Backtracking Search Optimization Algorithms Applied to Localization Problems. International Journal of Innovative Computing and Applications. 6(3-4) : 223-228, 2015.

[6] El HAmi A., RADi B., Uncertainty and optimization in structural mechanics, Wiley-ISTE, 2013.

[7] Patankar S.V., Numerical Heat Transfer and Fluid Flow, Hemisphere Publishing, New York, USA, 1980.

[8] Ferziger J.H., Peric M., Computational Methods for Fluid Dynamics, Springer, Berlin, Germany, 2002.

[9] Chopra A., Dynamics of Structures, Pearson Prentice Hall, 2nd ed, 2001.

[10] Bosman P.A.N., Thierens D., « The balance between proximity and diversity in multiobjective evolutionary algorithms ». IEEE Transactions on Evolutionary Computation, 7(2) : 174-188, 2003. 